\title{
Holes as Dipoles in a Doped Antiferromagnet and Stripe Instabilities
}

\author{
Su-Peng Kou ${ }^{a, b}$ and Zheng-Yu Weng ${ }^{a}$ \\ ${ }^{a}$ Center for Advanced Study, Tsinghua University, Beijing 100084 \\ ${ }^{b}$ Department of Physics, Beijing Normal University, Beijing, 100875
}

\begin{abstract}
Based on an effective model of a doped antiferromagnetic Mott insulator, we show that a doped hole will induce a dipole-like spin configuration in a spin ordered phase at low doping. The kinetic energy of doped holes is severely frustrated and a hole-dipole object is actually localized or selftrapped in space. Without a balance from the kinetic energy, the long-range dipole-dipole interaction between doped holes will dominate the low-energy physics, leading to an inhomogeneity instability as hole-dipoles collapse into stripes. Both antiphase metallic stripes of quarter-filling and antiphase insulating stripes along a diagonal direction are discussed as composed of hole-dipoles as elementary building blocks. Stripe melting and competing phases are also discussed.
\end{abstract}

PACS numbers: 74.20.Mn, 74.25.Ha, 75.10.-b

\section{INTRODUCTION}

The theoretical challenge of doped antiferromagnetic (AF) Mott insulator systems [1] comes from their nature of strong correlations. It makes the relevant models like the Hubbard and $t-J$ Hamiltonians highly difficult to deal with. In such systems, the kinetic energy of charge carriers usually gets strongly frustrated [2, 3] and there is no single dominant process based on which a perturbative approach can be constructed. This opens door for various competing orders at low doping and makes the phase diagram complex [4].

So far there still lacks a single microscopic description which can unify various competing phenomena known from both experiments in cuprates and theoretical considerations based on doped antiferromagnets. Given the fact that exact solutions of two-dimensional (2D) Hubbard and $t-J$ models are absent, a simplified and tractable model of doped AF Mott insulators is thus highly desirable in order to have a framework for a systematic study.

Such a type of models should incorporate three most essential ingredients of a doped AF Mott insulator. Namely, it should properly describes AF correlations at half-filling, including both long-range (low-energy) and short-range (high-energy) ones; Its Hilbert space should be restricted due to the presence of an upper Hubbard band at finite doping; Finally, it should explicitly embody essential frustration effects introduced by hole hopping in an antiferromagnet. Furthermore, compared to the original Hubbard and $t-J$ models, it ought to be simplified enough to allow a tractable treatment.

In this paper, we study an effective model [ [5, [6] of a doped AF-Mott insulator which satisfies the above criteria. This model well describes AF correlations at half-filling and precisely incorporates the so-called phase string effect at a finite doping. The latter has been identified [3] as a crucial dynamic frustration effect introduced by holes in the $t-J$ Hamiltonian. Here a phase string depicts a string of displaced transverse spins along a hole- hopping path, characterized by a sequence of signs:

$$
(+1) \times(-1) \times(-1) \times \cdots
$$

as illustrated by Fig. 1(a). It can be rigorously shown [3] that such transverse spin mismatches cannot be selfhealed simultaneously with the displaced spins in the longitudinal (quantization) direction [see Fig. 1(b)], through the superexchange term of the $t-J$ Hamiltonian. Consequently, after a hole moves through a closed path back to its origin and the displaced spins by hole hopping are restored in the quantization direction ( $\hat{z}$-axis), the wavefunction should always pick up an additional Berryphase-like sign given by (11), which is highly nontrivial in a general case.

Such a working model may be called the phase string model (section II). Based on it, we will show that in a spin ordered phase at low doping, a doped hole will generally induce a dipole-like spin configuration (section III). The kinetic energy of doped holes is severely frustrated by the phase string effect such that a hole-dipole is localized or self-trapped in space with an infinite mass. Without the balance from the kinetic energy, the long-range dipoledipole interaction between doped holes will dominate the low-energy physics. It will lead to an inhomogeneous instability as hole-dipoles collapse into stripes (section IV). These anti-phase stripes can be understood as that holes are threaded together by the phase strings. Both metallic stripes of anti-phase and quarter-filling and insulating stripe along the diagonal direction are discussed based on the elementary hole-dipoles. The stripe phase at low doping is highly competitive to homogeneous phases previously studied 7 in the same model, which include the AF long-range ordered state, spin glass state, and superconducting state. Therefore, a rich array of phases are revealed in the phase string model, where AF correlations and phase string effect serve as basic driving forces. 


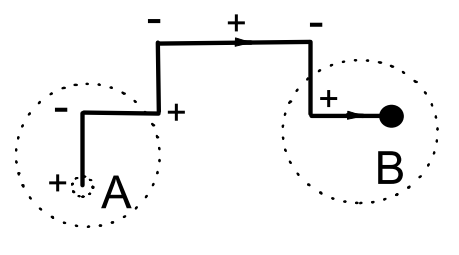

transverse components

(a)

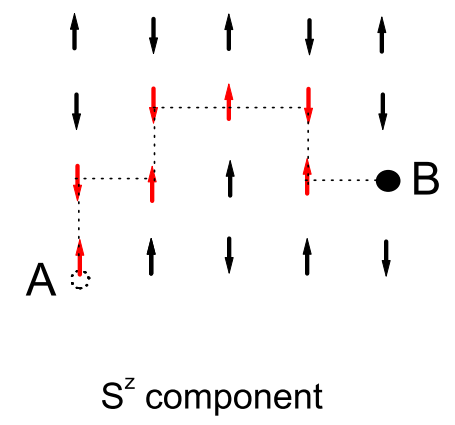

(b)

FIG. 1: Spin mismatches created by a hole hopping in a Néel order: (a) in transverse directions (phase string) and (b) in longitudinal direction. Two dotted circles in (a) indicate the sigularity of the starting and ending points in a holon path (see text).

\section{AN EFFECTIVE MODEL OF A DOPED ANTIFERROMAGNET}

The $t-J$ Hamiltonian is one of the simplest nontrivial models of doped Mott insulator/doped antiferromagnets [1]. Based on the exact phase string formulation [5] of the $t-J$ Hamiltonian, an effective low-energy model can be derived, known as the phase string model 6 .

The presence of short-range AF correlations provides a natural "ultraviolet" cutoff in deducing such an effective model from the $t-J$ Hamiltonian. So the local AF correlations, characterized by the bosonic RVB pairing 8], underpin this effective theory. It differs from the slaveboson mean-field theories [9] by emphasizing the underlying AF correlations; It differs from the slave-fermion mean-field theories [10, 11] by precisely dealing with a singular hopping effect in the $t-J$ model, i.e., the phase string effect mentioned in Introduction.

Such an effective model itself defines a class of doped Mott-insulator/doped antiferromagnet. It has been shown to possess the following properties: (I) It well describes antiferromagnetism at half-filling, including both long-wavelength, low-energy and short-range, highenergy (high-temperature) physics; (III) It has a d-wave superconducting ground state beyond a finite doping concentration; (II) It characterizes the correct restricted Hilbert space for charge and spin degrees of freedom of a doped Mott insulator, when holes are introduced.

The effective Hamiltonian can be written [5, 6] as $H_{\text {string }}=H_{h}+H_{s}$, where

$$
\begin{aligned}
& H_{h}=-t_{h} \sum_{\langle i j\rangle}\left(e^{i A_{i j}^{s}-i \phi_{i j}^{0}}\right) h_{i}^{\dagger} h_{j}+H . c . \\
& H_{s}=-J_{s} \sum_{\langle i j\rangle \sigma}\left(e^{i \sigma A_{i j}^{h}}\right) b_{i \sigma}^{\dagger} b_{j-\sigma}^{\dagger}+H . c .
\end{aligned}
$$

with $h_{i}^{\dagger}$ and $b_{i \sigma}$ denoting bosonic holon and spinon operators, respectively. When the holon number is zero, $H_{s}$ is equivalent to the Schwinger-boson mean-field Hamiltonian [10], which well describes AF correlations at halffilling. The AF long range order is realized by the spinon Bose condensation, i.e., $\left\langle b_{i \sigma}\right\rangle \neq 0$ at zero temperature. At larger doping, when holons are Bose condensed, the ground state becomes a (d-wave) superconductor 12].

A unique feature of this effective model is the presence of link fields in Eqs. (2) and (3). They are not free gauge fields as they satisfy the topological constraints

$$
\sum_{c} A_{i j}^{h}= \pm \pi \sum_{l \in c} n_{l}^{h}
$$

and

$$
\sum_{c} A_{i j}^{s}= \pm \pi \sum_{l \in c}\left(n_{l \uparrow}^{b}-n_{l \downarrow}^{b}\right),
$$

for a closed loop $c$, in which $n_{l}^{h}$ and $n_{l \alpha}^{b}$ are holon and spinon number operators, respectively. In addition to $A_{i j}^{s}, \phi_{i j}^{0}$ in Eq. (2) describes a uniform $\pi$ flux per plaquette $\left(\sum_{\square} \phi_{i j}^{0}= \pm \pi\right)$. This model has a $\mathrm{U}(1) \times \mathrm{U}(1)$ gauge symmetry as $H_{h}$ and $H_{s}$ are invariant under the gauge transformations:

$$
h_{j} \rightarrow h_{j} \exp \left(i \varphi_{j}\right), A_{i j}^{s} \rightarrow A_{i j}^{s}+i\left(\varphi_{i}-\varphi_{j}\right),
$$

and

$$
b_{j \sigma} \rightarrow b_{j \sigma} \exp \left(i \sigma \phi_{j}\right), A_{i j}^{h} \rightarrow A_{i j}^{h}+i\left(\phi_{i}-\phi_{j}\right) .
$$


Here the holons and spinons are basic building blocks that properly characterize the restricted Hilbert space of a doped Mott insulator. But it is important to note that they, as independent entities, are not necessarily the true low-lying elementary excitations of the system. In this model, the electron operator is made of holon and spinon operators in the following form [5]

$$
c_{i \sigma}=h_{i}^{\dagger} b_{i \sigma} e^{i \hat{\Theta}_{i \sigma}},
$$

where $\hat{\Theta}_{i \sigma}$ is a topological phase, which ensures the fermionic statistics of $c_{i \sigma}$, as defined by

$$
e^{i \hat{\Theta}_{i \sigma}}=(-\sigma)^{i} e^{\frac{i}{2}\left[\Phi_{i}^{b}-\sigma \Phi_{i}^{h}\right]},
$$

with

$$
\Phi_{i}^{b}=\sum_{l \neq i} \operatorname{Im} \ln \left(z_{i}-z_{l}\right)\left(\sum_{\alpha} \alpha n_{l \alpha}^{b}-1\right),
$$

and

$$
\Phi_{i}^{h}=\sum_{l \neq i} \operatorname{Im} \ln \left(z_{i}-z_{l}\right) n_{l}^{h} .
$$

Thus, going from the holon-spinon representation to the electron representation, topological (vortexlike) phases, $\Phi_{i}^{b}$ and $\Phi_{i}^{h}$, are involved, which implies that the holon and spinon objects defined in the phase string model are topological entities themselves, as will soon become clear below.

\section{DOPED HOLES: DIPOLES IN SPIN ORDERED PHASE}

How doped holes propagate in an antiferromagnet and how background spins are influenced by the hole motion are two central issues in the study of doped antiferromagnets. In the present section, we will focus on these issues in a spin ordered phase at small doping, based on the above phase string model.

\section{A. Holons as merons}

The spin-flip operator can be expressed by

$$
S_{i}^{+}=(-1)^{i} b_{i \uparrow}^{\dagger} b_{i \downarrow} \exp \left[i \Phi_{i}^{h}\right],
$$

according to Eq. (8). The presence of the vortex phase $\Phi_{i}^{h}$ in Eq. (12) clearly shows that a holon is a topological object that affects spins nonlocally.

At half-filling where $\Phi_{i}^{h}=0$, the Bose condensation $<$ $b_{i \sigma}>\neq 0$ of spinons gives rise to an AF long-range order of spins lying in the x-y plane: $\left.\left\langle S_{i}^{+}\right\rangle=(-1)^{i}<b_{i \uparrow}^{\dagger}\right\rangle<$ $b_{i \downarrow}>$. Through $\Phi_{i}^{h}$, each holon will induce a "meron" spin twist, which holds true as long as $\left\langle b_{i \sigma}>\neq 0\right.$. Such
FIG. 2: A meron-like spin configuration around a holon.

a spin vortex configuration (for a holon at the origin) may be characterized by the unit vector

$$
\mathbf{n}_{i} \propto(-1)^{i}\left\langle\mathbf{S}_{i}\right\rangle,
$$

as follows:

$$
\mathbf{n}_{i}=\mathbf{r}_{i} /\left|\mathbf{r}_{i}\right|,
$$

with $\mathbf{r}_{i}^{2}=x_{i}^{2}+y_{i}^{2}$. It is schematically shown in Fig. 2 .

To evaluate the potential energy of a holon (meron), we consider the spinon condensed part in $H_{s}$ [Eq. [3] ]:

$$
\Delta E_{s} \simeq-J_{s} \rho_{c}^{s} a^{2} \sum_{\langle i j\rangle}\left[\cos A_{i j}^{h}-1\right]+\text { c.c. }
$$

in which $\rho_{c}^{s} a^{2} \equiv<b^{\dagger}>^{2}(a$ is the lattice constant) and a reference energy corresponding to $A_{i j}^{h}=0$ has been subtracted. Explicitly $A_{i j}^{h}$ reads

$$
A_{i j}^{h}=\frac{1}{2} \sum_{l \neq i, j} \operatorname{Im} \ln \frac{z_{i}-z_{l}}{z_{j}-z_{l}} n_{l}^{h}
$$

and then one has

$$
\begin{aligned}
\Delta E_{s} & \simeq J_{s} \rho_{c}^{s} a^{2} \sum_{\langle i j\rangle}\left(A_{i j}^{h}\right)^{2} \\
& \equiv E_{s}^{m}+E_{s}^{m-m}
\end{aligned}
$$

where $E_{s}^{m}=\sum_{l} n_{l}^{h} \varepsilon^{m}$ with a single holon-meron selfenergy

$$
\begin{aligned}
\varepsilon^{m} & \equiv \frac{J_{s} \rho_{c}^{s} a^{2}}{4} \sum_{\langle i j\rangle(\neq l)}\left(\operatorname{Im} \ln \frac{z_{i}-z_{l}}{z_{j}-z_{l}}\right)^{2} \\
& \simeq \frac{\pi}{2} J_{s} \rho_{c}^{s} a^{2} \ln (L / a),
\end{aligned}
$$


where $L$ is the size of the sample. Namely, a single holon as a meron in the spinon condensed phase will generally cost a logarithmically divergent energy.

Furthermore, the meron-meron interaction is given by

$$
E_{s}^{m-m}=\sum_{l<l^{\prime}} n_{l}^{h} V_{l l^{\prime}}^{m-m} n_{l}^{h},
$$

and

$$
\begin{aligned}
V_{l l^{\prime}}^{m-m} & =\frac{J_{s} \rho_{c}^{s} a^{2}}{2} \sum_{\langle i j\rangle\left(\neq l l^{\prime}\right)} \operatorname{Im} \ln \frac{z_{i}-z_{l}}{z_{j}-z_{l}} \operatorname{Im} \ln \frac{z_{i}-z_{l^{\prime}}}{z_{j}-z_{l^{\prime}}} \\
& \simeq-\pi J_{s} \rho_{c}^{s} a^{2} \ln \frac{\left|\mathbf{r}_{l}-\mathbf{r}_{l^{\prime}}\right|}{L} .
\end{aligned}
$$

Here two holons will always repulse each other logarithmically, as they are merons of the same topological charge.

We note that there have been proposals of hole-merons in literature [13, 14]. They usually involve both merons and anti-merons. Two holes may thus be bound like a meron-anti-meron (vortex-antivortex) pair. By contrast, in the present case holons are always repulsive to each other as they carry the same topological charge. The underlying physical reason is that this is in favor of the kinetic energy for a finite concentration of holons, which become identical bosons after explicitly incorporating the phase string effect in the slave-fermion formalism. Consequently, superconductivity in the present doped AF-Mott insulator originates 12] from the Bose condensation of holons, instead of the pairing of them. Here one should distinguish the holon pairing from the pairing order parameter of electrons. The latter still characterizes the superconducting state in the present case where spins are in RVB pairing, even though the holon pairing is absent.

Intuitively one might envisage that a phase string induced by one hole be "erased" by an another hole accompanying it. This may look like in favor of hole pairing. But such a picture is actually more in favor of stripes in which many holes precisely follow each other's paths to eliminate the phase string effect. The basic reason is that the phase string effect of (1) is not simply a "geometric" effect: it also involves spin dynamics and is very singular. Thus, unless one hole tightly follows the other along the same path, like in the stripe case, the singular dynamic phase string effect cannot be completely removed by two holes simply staying close to each other. In fact, previously Trugman [2, 4] has already found the presence of frustrations for a hole pair's motion in an antiferromagnet, due to a quantum effect originating from the fermionic character of the background spins. This effect is actually included in the phase string effect in the present approach. Finally, we note that technically, a holon is just a building block in the phase string model and the possibility that some holons may be anti-merons can be, in principle, constructed as composite objects in the present framework. But such a construction is usually not energetically favorable at finite doping and will

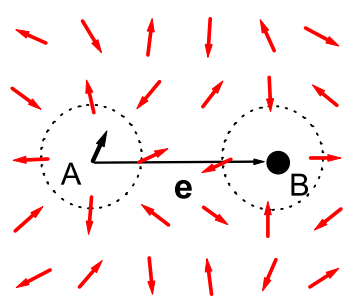

FIG. 3: A hole-dipole is a confined object composed of a holon-meron and an anti-meron at two poles: B and A, connected by a branch-cut with the spatial separation $\mathbf{e}$ as a dipole moment.

be discussed elsewhere. The pairing of two doped holes has been discussed [15] in the $t-J_{z}$ model based on the "string" picture before.

\section{B. Holon confinement: Doped holes as dipoles}

To remove such an energy divergence [Eq.(17)] associated with a meron spin configuration, each holon will have to "nucleate" an anti-meron from the vacuum. Being bound to the latter, a dipole composite with a finite energy will be formed. This elementary object will carry a charge $+e$ and replace the holon to represent a true stable hole in the spin ordered phase. By contrast, free holons disappear from the finite-energy spectrum. We can also call this phase as the holon confined phase.

\section{Formation of dipoles}

An anti-meron configuration is defined as a spin twist

$$
b_{i \sigma} \rightarrow \tilde{b}_{i \sigma} \exp \left[i \frac{\sigma}{2} \vartheta_{i}^{k}\right]
$$

where $\vartheta_{i}^{k}=\operatorname{Im} \ln \left(z_{i}-z_{k}^{0}\right)$. Here $z_{k}^{0}$ denotes the position (center) of an anti-meron labeled by $k$. Correspondingly

$$
\left\langle S_{i}^{+}\right\rangle \rightarrow(-1)^{i}\left\langle\tilde{b}_{i \uparrow}^{\dagger}\right\rangle\left\langle\tilde{b}_{i \downarrow}\right\rangle \exp \left[i \Phi_{i}^{h}-i \Omega_{i}\right]
$$

where $\Omega_{i}=\sum_{k} \vartheta_{i}^{k}$ (summing over all induced antimerons). If $z_{k}^{0}$ 's are close to the positions of correspond- 
ing holons, then cancellation occurs in $\Phi_{i}^{h}-\Omega_{i}$. Consequently, the logarithmic divergence is removed from $H_{s}$ (see below).

Define $n_{i}^{x}+i n_{i}^{y}=e^{i \phi_{0}+i \phi_{i}}$, with the unit vector $\mathbf{n}_{0} \equiv$ $\left(\cos \phi_{0}, \sin \phi_{0}\right)$ as the magnetization direction at infinity. For a bound pair of meron (holon)-anti-meron centered at the origin, labeled by the index $k$, one obtains according to (21)

$$
\phi_{i}^{k}=\operatorname{Im} \ln \frac{z_{i}-z_{k} / 2}{z_{i}+z_{k} / 2},
$$

with $z_{k} \equiv e_{k}^{x}+i e_{k}^{y}$. Here $\mathbf{e}_{k}$ denotes the spatial displacement of the meron and anti-meron centered at $\pm \frac{\mathbf{e}_{k}}{2}$, respectively. At $\left|\mathbf{r}_{i}\right|>>\left|\mathbf{e}_{k}\right|$,

$$
\phi_{i}^{k} \simeq \frac{\left(\hat{\mathbf{z}} \times \mathbf{e}_{k}\right) \cdot \mathbf{r}_{i}}{\left|\mathbf{r}_{i}\right|^{2}} .
$$

Thus, each pair of holons and anti-merons forms a composite, as shown in Fig. 3, that has a dipolar spin configuration at a sufficiently large distance.

In $H_{s}$, one may easily check that $\omega_{i j} \equiv\left(\Omega_{i}-\Omega_{j}\right) / 2$ cancels out $A_{i j}^{h}$ and thus removes the aforementioned energy divergence. Here the energy cost Eq. (14) is replaced by

$$
\begin{aligned}
\Delta E_{s} & =-2 J_{s} \rho_{c}^{s} a^{2} \sum_{\langle i j\rangle}\left[\cos \left(A_{i j}^{h}-\omega_{i j}\right)-1\right] \\
& \simeq J_{s} \rho_{c}^{s} a^{2} \sum_{\langle i j\rangle}\left(A_{i j}^{h}-\omega_{i j}\right)^{2} \\
& \equiv \sum_{k} \mathcal{E}_{k}^{d}+\sum_{k<k^{\prime}} V_{k k^{\prime}}^{d-d},
\end{aligned}
$$

in which the single dipole energy

$$
\mathcal{E}_{k}^{d}=\frac{J_{s} \rho_{c}^{s} a^{2}}{4} \sum_{\langle i j\rangle}\left(\phi_{i}^{k}-\phi_{j}^{k}\right)^{2},
$$

and dipole-dipole interaction energy

$$
V_{k k^{\prime}}^{d-d}=\frac{J_{s} \rho_{c}^{s} a^{2}}{2} \sum_{\langle i j\rangle}\left(\phi_{i}^{k}-\phi_{j}^{k}\right)\left(\phi_{i}^{k^{\prime}}-\phi_{j}^{k^{\prime}}\right) .
$$

In the continuum limit, $\phi_{i}^{k}-\phi_{j}^{k} \approx\left(\mathbf{r}_{i}-\mathbf{r}_{j}\right) \cdot \nabla \phi^{k}$ and, according to Eq. (22),

$$
\nabla \phi^{k}=\frac{\hat{\mathbf{z}} \times\left(\mathbf{r}-\mathbf{e}_{k} / 2\right)}{\left|\mathbf{r}-\mathbf{e}_{k} / 2\right|^{2}}-\frac{\hat{\mathbf{z}} \times\left(\mathbf{r}+\mathbf{e}_{k} / 2\right)}{\left|\mathbf{r}+\mathbf{e}_{k} / 2\right|^{2}} .
$$

Then the self-energy of a dipole can be evaluated by $\mathcal{E}_{k}^{d} \simeq$ $\frac{J_{s} \rho_{c}^{s} a^{2}}{4} \int d^{2} \mathbf{r}\left(\nabla \phi^{k}\right)^{2}$. In contrast to the logarithmically divergent meron-energy, there is a branch-cut connecting two poles at $\pm \frac{\mathbf{e}_{k}}{2}$ of the hole dipole, and $\mathcal{E}_{k}^{d}$ becomes finite

$$
\begin{aligned}
\mathcal{E}_{k}^{d} & \simeq \frac{J_{s} \rho_{c}^{s} a^{2}}{4} \int d^{2} \mathbf{r} \frac{\left|\mathbf{e}_{k}\right|^{2}}{\left|\mathbf{r}-\mathbf{e}_{k} / 2\right|^{2}\left|\mathbf{r}+\mathbf{e}_{k} / 2\right|^{2}} \\
& \simeq \pi J_{s} \rho_{c}^{s} a^{2} \ln \frac{\left|\mathbf{e}_{k}\right|+a}{a},
\end{aligned}
$$

at $\left|\mathbf{e}_{k}\right| \gtrsim a$.

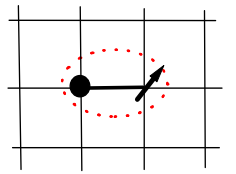

Type A dipole

(a)

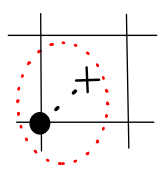

Type B dipole
FIG. 4: Two typical hole-dipoles of minimal size: (a) Type A dipole with a holon and a spin sitting at two poles at nearestneighboring lattice sites; (b) Type B dipole with one pole at the center of a plaquette.

\section{Type A and Type B dipoles}

Let us first point out that generally there are two possible anti-meron configurations as defined by Eq. (20). Type A: the core sits on a lattice site $l$, i.e., $z_{k}^{0}=z_{l}$. In this case, there will be always a spin $1 / 2$ sitting at the core of the anti-meron [Fig. 3], according to the singleoccupancy constraint. Type B: $z_{k}^{0}$ is located inside a lattice plaquette, instead of a lattice site. Figs. 4(a) and (b) illustrates both cases at minimal sizes.

Type A dipole. Besides the energy consideration given above, it is important to make sure the self-consistency of the theory, that is, the anti-meron configuration Eq. (20) should not violate the single-valueness of the electron operator (the spin operator in Eq. (21) is obviously single-valued).

Consider that a winding of the coordinate $z_{i}$ around $z_{k}^{0}$ which is located at a lattice site. A phase $\pm \pi$ (corresponding to $\sigma= \pm 1$ ) will be acquired on the r.h.s. of Eq. (20). In order to ensure the single-valueness of $c_{i \sigma}$, the holon field has to be transformed in response to Eq. (20). We may introduce

$$
h_{i}^{\dagger} \rightarrow h_{i}^{\dagger} e^{-i \frac{\alpha_{k}}{2} \operatorname{Im} \ln \left(z_{i}-z_{k}^{0}\right)},
$$

with the spin index $\alpha_{k}= \pm 1$ denoting the spin at the site $z_{k}^{0}$. Then the total change in the electron operator $c_{i \sigma}$ (for a winding of the coordinate $z_{i}$ around $z_{k}^{0}$ ) will be $e^{i\left(\sigma \pi-\alpha_{k} \pi\right)}=1$, still satisfying the single-valueness condition. 
The physical implication of (29) is as follows. It will change $H_{h}$ in Eq. (2) by

$$
A_{i j}^{s} \rightarrow \tilde{A}_{i j}^{s} \equiv A_{i j}^{s}-\frac{\alpha_{k}}{2} \operatorname{Im} \ln \frac{z_{i}-z_{k}^{0}}{z_{j}-z_{k}^{0}} .
$$

It simply removes the contribution of the spinon, $\sum_{\sigma} \sigma n_{l \sigma}^{b} \equiv \alpha_{k}$, at the site of $z_{l}=z_{k}^{0}$ from $A_{i j}^{s}$, i.e.,

$$
\sum_{c} \tilde{A}_{i j}^{s}=\sum_{c} A_{i j}^{s}-\alpha_{k} \pi
$$

where $c$ is a loop encircling $z_{k}^{0}$. By incorporating the phase on the r.h.s. of Eq. (29) into $\hat{\Theta}_{i \sigma}$ of Eq. (8), one may also see that the contribution from the spinon at the site of $z_{k}^{0}$ is eliminated from $\Phi_{i}^{b}$ and thus from $c_{i \sigma}$. It means that the spin at $z_{k}^{0}$ will no longer exert the nonlocal effect, embedded in a spinon, through $A_{i j}^{s}$ in $H_{h}$ and $\hat{\Theta}_{i \sigma}$ in $c_{i \sigma}$. Hence, to maintain the single-valueness condition after the creation of an anti-meron, the spin 1/2 at its center site $z_{k}^{0}$ will lose its spinon character and looks like a local "defect" spin.

Such a "defect" spin, sitting at the core of an antimeron, will be decoupled from the environmental spins and simply behave like a free $S=1 / 2$ spin. This can be straightforwardly checked. By noting that the transformation Eq. (20) excludes the case $z_{i}=z_{k}^{0}$ due to the uncertainty of $\vartheta_{i}^{k}$, the phases of those terms in $H_{s}$ that involve the links attached to the site $z_{k}^{0}$, will become uncertain after the transformation. Specifically, these terms connected to $z_{k}^{0}$ will all vanish when one averages over $\vartheta_{0}$ after the replacement $\vartheta_{i}^{k} \rightarrow \vartheta_{i}^{k}+\vartheta_{0}$ in Eq. 201 for $z_{i} \neq z_{k}^{0}$, while the rest of $H_{s}$ remains invariant. Thus, the spin $1 / 2$ at $z_{k}^{0}$ is indeed decoupled from the environmental spins.

Type $B$ dipole. Now consider the case that $z_{k}^{0}$ is located at a center of a plaquette [see Fig. 4(b)]. There is no longer a lattice spin at $z_{k}^{0}$ and the holon transformation Eq. (29) may be replaced by the form

$$
h_{i}^{\dagger} \rightarrow h_{i}^{\dagger} e^{ \pm i \frac{1}{2} \vartheta_{i}^{k}} .
$$

One can check that $c_{i \sigma}$ still remains single-valued for a winding of the coordinate $z_{i}$ around $z_{k}^{0}$. Based on Eq. (32), the holon Hamiltonian $H_{h}$ is changed by $A_{i j}^{s} \rightarrow \tilde{A}_{i j}^{s}$, with

$$
\tilde{A}_{i j}^{s} \equiv A_{i j}^{s} \pm \frac{1}{2} \operatorname{Im} \ln \frac{z_{i}-z_{k}^{0}}{z_{j}-z_{k}^{0}} .
$$

Physically it means that an additional $\pm \pi$ flux penetrates through the plaquette where $z_{k}^{0}$ resides:

$$
\sum_{c} \tilde{A}_{i j}^{s}=\sum_{c} A_{i j}^{s} \pm \pi
$$

with $c$ as a loop encircling the given plaquette.

In contrast to Type A, it is not obvious that there is a free spin $1 / 2$ trapped around the plaquette of the core of the anti-meron. In Type B, the cost of the superexchange energy is mainly due to the spin twist around the plaquette $z_{k}^{0}$ which is less dramatic than in Type A, where the presence of an isolated spin $1 / 2$ at $z_{k}^{0}$ means the loss of the superexchange energy for four nearest-neighboring links connected to it. But in Type B, there are additional $\pi$ flux quanta [see Eq. (34)] threading through the plaquettes, where the cores of anti-merons are located, seen by holons. So the hopping energy of holons in Type B is different from that of a Type A dipole. Generally, Type A dipoles are expected to be more stable at higher doping, while Type B dipoles should be energetically favorable at low doping.

\section{Localization of hole dipoles and phase string effect}

The fact that a doped hole will induce a dipolar spin configuration was first pointed out by Shraiman and Siggia [16] based on a semi-classical treatment of the $t-J$ model. In Shraiman-Siggia's picture, the hole is sitting at the center of the dipole and such a hole-dipole is mobile in space. This mobility may be easily understood if one considers the translational invariance and the fact that the hopping overlap integral for two hole-dipoles with their centers displaced by a lattice constant should be finite in general. That a mobile hole carries a long-range dipolar spin distortion has been also studied in Refs. [17, 18].

By contrast, in the present case, the hole is sitting at one of the two opposite poles of a dipole, not at its center. The holon itself has a finite hopping integral in $H_{h}$ and can hop around. Nonetheless it is confined to an antimeron by a logarithmic potential in Eq. (28). On the other hand, an anti-meron itself as a semi-classical vortex formed by the condensed spinons is immobile: it cannot propagate in terms of $H_{s}$. In other words, its effective mass is infinite, which is in contrast to a holon which has a finite hopping integral, or mass, according to $H_{h}$. Therefore, the hole dipole (both Type A and B) as a whole must remain localized or self-trapped in space.

This distinction between the Shraiman-Sigga dipole and the present one can be traced back to a very singular effect in the $t-J$ model, which has been inappropriately treated in the semi-classical approach of Shraiman-Sigga. In the following we further clarify this issue.

According to Eq. (22), there is a branch-cut connecting two poles (at $\pm \frac{\mathbf{e}_{k}}{2}$, respectively) of a hole dipole at the origin, as illustrated in Fig. 3. Such a branch-cut can be physically understood in terms of the irreparable phase string effect, which was discovered [3] as a generic property of the $t-J$ model.

A phase string is basically a sequence of + and - signs shown in (1) [also see Fig. 1(a)], which is left by a spinless hole on the path as it moves on a spin background described by the $t-J$ model. The origin of the phase string is due to the disordered Marshall sign caused by the hole hopping [3]. Physically it may be interpreted as describing displaced spins, by hopping, along the trans- 
verse directions (in $\mathrm{x}-\mathrm{y}$ plane). Specifically, the sign + and - in (1) distinguish the "backflow" spin $\uparrow$ and $\downarrow$ exchanged with a hole at each step of hopping. Since the superexchange term "respects" the Marshall sign, the phase string, describing the displaced Marshall sign, cannot be "repaired" through the superexchange term. Therefore, if one draws a circle around either the hole at B or the starting point at A, it will always intercept the phase string once (or odd times) [see Fig. 1(a)].

Pictorially, one may consider a bare hole created by the electron $c$-operator at a point $\mathrm{A}$ and then imagine that the spinless hole (holon) hops to the point B. It results in a dipole configuration with the two poles as its starting point $\mathrm{A}$ and ending point $\mathrm{B}$, connected by the phase string in between [Fig. 1(a)]. Since the holon can reach $B$ from different paths originated at $A$, the singular phase string is then replaced by (or relaxes to) a smoother dipole configuration. Nonetheless, the topology remains as represented by the branch-cut connecting two poles of the dipole as illustrated by Fig. 3.

In a Néel state with the magnetization lying in $\mathrm{x}-\mathrm{y}$ plane, the displacement of staggered magnetization moments will reach the maximum, namely, $\pi$ phase per site (inversion of the spin polarization) on the hole path. It implies that going through the circle once, around the point A or B in Fig. 1(a), one finds the total spin displacement relative to the original Néel spin background is $\pi$, modulo $2 \pi$, which has the same topology as a meron or anti-meron discussed above.

Finally, the localization of a hole dipole can be physically understood in terms of the phase string picture too [19]. Since the phase string (1) cannot be "self-healed" through, say, spin dynamics, a holon moves far away from its origin A will eventually lose its phase coherence as the product (1) becomes uncertain due to the zero-point spin fluctuations in the Néel background. It means the propagating amplitude vanishes such that the holon must be localized or self-trapped around the origin A within a finite distance. The single electron spectral function for the one-hole doped case has been studied [19] based on such a holon localization description, in which the dispersion of the low-lying peak is originated from the "spinon" sepectrum instead. The calculated spectral function well explains the corresponding photoemission experiments [19].

Delocalization of holons will occur at higher doping when the spin background is changed (self-consistently) by the phase string effect. Such a holon deconfined phase is generally a superconducting state as discussed in Refs. 7, 12 On the other hand, at low doping, without the destruction of spin ordering, delocalization of holons can only be realized in the reduced dimension when holons follow each other's paths such that the induced phase string frustrations can be eliminated. Such a metallic stripe in the spinon condensed phase will be discussed in the following section.

\section{STRIPE INSTABILITY}

As discussed above, a hole-dipole object does not have a kinetic energy, or, its self-energy does not have a dispersion, since it is self-trapped in space with an infinite mass. Thus the long-range dipole-dipole interaction among dipoles may become crucial in determining the low-energy physics in such a phase where kinetic energy is strongly frustrated. In this part we will discuss the origin of stripes based on this picture.

\section{A. Dipole-dipole interaction}

For a dipole located at the origin, Eq. 27) can be reduced to

$$
\nabla \phi^{k} \simeq \frac{1}{|\mathbf{r}|^{2}}\left[2 \frac{(\hat{\mathbf{z}} \times \mathbf{r})\left(\mathbf{r} \cdot \mathbf{e}_{k}\right)}{|\mathbf{r}|^{2}}-\hat{\mathbf{z}} \times \mathbf{e}_{k}\right],
$$

for $|\mathbf{r}|>>\left|\mathbf{e}_{k}\right|$ and a singular part

$$
\nabla \phi^{k} \simeq-2 \pi\left(\hat{\mathbf{z}} \times \mathbf{e}_{k}\right) \delta(\mathbf{r})
$$

at $|\mathbf{r}| \sim 0\left(\left|\mathbf{e}_{k}\right| \rightarrow 0\right)$.

Then, in terms of (26), the dipole-dipole interaction between $k$-th and $k^{\prime}$-th dipoles, located at $\mathbf{r}_{k}$ and $\mathbf{r}_{k^{\prime}}$, respectively, which are well-separated in space by $\left|\mathbf{r}_{k k^{\prime}}\right| \equiv$ $\left|\mathbf{r}_{k}-\mathbf{r}_{k^{\prime}}\right| \gg\left|\mathbf{e}_{k}\right|$, can be expressed in leading order approximation by

$$
\begin{aligned}
V_{k k^{\prime}}^{d-d} & =\frac{J_{s} \rho_{c}^{s} a^{2}}{2} \int d^{2} \mathbf{r} \nabla \phi^{k} \cdot \nabla \phi^{k^{\prime}} \\
& \simeq-J_{s} \rho_{c}^{s} a^{2} \pi\left[\left(\hat{\mathbf{z}} \times \mathbf{e}_{k}\right) \cdot \nabla \phi^{k^{\prime}}\left(\mathbf{r}_{k}\right)-\left(k \leftrightarrow k^{\prime}\right)\right] \\
& \left.\simeq \frac{2 \pi J_{s} \rho_{c}^{s} a^{2}}{\left|\mathbf{r}_{k k^{\prime}}\right|^{2}}\left[\mathbf{e}_{k} \cdot \mathbf{e}_{k^{\prime}}-2 \frac{\left(\mathbf{e}_{k} \cdot \mathbf{r}_{k k^{\prime}}\right)\left(\mathbf{e}_{k^{\prime}} \cdot \mathbf{r}_{k k^{\prime}}\right)}{\left|\mathbf{r}_{k k^{\prime}}\right|^{2}}\right] 7\right)
\end{aligned}
$$

Here $V_{k k^{\prime}}^{d-d}$ has a typical form of magnetic dipole-dipole interaction, with $\mathbf{e}_{k}$ denoting the dipole moment.

Consider orientations of dipole moments which minimize the energy of the dipole-dipole interaction Eq. (37) at a fixed distance $\left|\mathbf{r}_{k k^{\prime}}\right|$. Define $\cos \varphi_{k}=\mathbf{e}_{k}$. $\mathbf{r}_{k k^{\prime}} /\left|\mathbf{e}_{k}\right|\left|\mathbf{r}_{k k^{\prime}}\right|$ and $\cos \varphi_{k^{\prime}}=\mathbf{e}_{k^{\prime}} \cdot \mathbf{r}_{k k^{\prime}} /\left|\mathbf{e}_{k^{\prime}}\right|\left|\mathbf{r}_{k k^{\prime}}\right|$. We have

$$
V_{k k^{\prime}}^{d-d}=-\frac{2 \pi J_{s} \rho_{c}^{s} a^{2}\left|\mathbf{e}_{k}\right|\left|\mathbf{e}_{k^{\prime}}\right|}{\left|\mathbf{r}_{k k^{\prime}}\right|^{2}} \cos \left(\varphi_{k}+\varphi_{k^{\prime}}\right),
$$

which is minimized at

$$
\varphi_{k}=-\varphi_{k^{\prime}}+\text { modulo } 2 \pi
$$

with

$$
V_{\text {min }}^{d-d}=-\frac{2 \pi J_{s} \rho_{c}^{s} a^{2}\left|\mathbf{e}_{k}\right|\left|\mathbf{e}_{k^{\prime}}\right|}{\left|\mathbf{r}_{k k^{\prime}}\right|^{2}}
$$

Other orientations of dipole moments always give rise to higher energies for a given $\left|\mathbf{r}_{k k^{\prime}}\right|$. For instance, it is easy to see that for the case of random orientations $<$ $\left.\mathbf{e}_{k} \cdot \mathbf{e}_{k^{\prime}}\right\rangle=0,\left\langle V_{k k^{\prime}}^{d-d}\right\rangle=0$. 


\section{B. Stripe instability}

Due to translational invariance, the center of a holedipole can be located at any spatial point with the same self-energy. Thus, to minimize (40), the distance $\left|\mathbf{r}_{k k^{\prime}}\right|$ between two dipoles should be continuously shrunk. Without a balance from the kinetic energy, such collapsing seems an inevitable consequence of the dipole-dipole interaction. Of course, single hole-dipole picture is only meaningful at a scale $\left|\mathbf{r}_{k k^{\prime}}\right| \gtrsim\left|\mathbf{e}_{k}\right|$. Below such a scale, one has to take into account the detailed local physics to determine the hole-spin configuration and energy.

It is important to note that this dipole-dipole "attraction" does not simply result in a real-space pairing of holes. Due to the lack of kinetic energy cost, more dipoles would rather like to lump together, with orientations of dipole moments satisfying Eq. (39), in order to lower the potential energy. They can thus either line up to form a stripe or phase separate into Wigner-crystal-like clusters. In reality, the existence of long-range Coulomb interaction may generally favor [4] a stripe configuration over large Wigner-crystal-like clusters. Moreover, the latter possibility can be also considered as a further instability of stripes when they are attractive to each other and subsequently collapse into $2 \mathrm{D}$ clusters. Thus one can always focus on single stripes first, based on the hole-dipole picture. In fact, we shall see below that, for instance, forming a metallic stripe will lead to a disassociation of individual dipoles and release additional kinetic energies which can further stabilize stripes against forming a $2 \mathrm{D}$ cluster.

One possible loophole in the above argument is the existence of some other forces which may be in competition with the dipole-dipole interaction. We first note that the dipole-dipole interaction could be quite weak. In a holedipole shown in Fig. 3, the holon sitting at one pole is actually not static: it generally will hop around the pole at $\mathrm{A}$, in terms of $H_{h}$ [Eq. (2)], such that $\left\langle\mathbf{e}_{k}\right\rangle$ may get strongly suppressed, and so does the dipole-dipole interaction. It is a matter of competition between the hopping energy of holons (which contributes to the self-energy of a hole-dipole) and the dipole-dipole interaction in Eq. (38) or Eq. (40) that self-consistently determines the actual dipole moment $\left\langle\mathbf{e}_{k}\right\rangle$.

In realistic situation, for example, a small amount of disorders can easily pin down hole-dipoles without the penalty from kinetic energy, such that the distribution of holes may still remain uniform on average. Based on a homogeneous picture, it has been previously shown [7] that the AF long range state will persist over some finite doping concentration if interlayer spin-spin coupling is considered in the same model. The dipole-dipole interaction will then be responsible for causing a cluster spin glass phase beyond some critical concentration of holes and eventually an insulating-superconducting transition takes place at a critical doping $x_{c} \sim 0.043$ []].

Even as a metastable state with local minima, such uniform phases may only evolve into stripe states of lower energy after a macroscopic time scale. So these uniform insulating phases are highly competitive with the inhomogeneous phases, in a real experimental situation where a quenched disordered phase from high temperature may result in a spin glass phase [7, 20, 21, 22, 23], with the help of impurities, instead of a more ordered stripe phase at low temperature.

\section{Static stripes}

So the long-range dipole-dipole interaction will be responsible for doped holes to "talk" to each other and for them to collectively collapse into stripes. It provides a mechanism for the instability of a homogeneity insulating phase and the formation of stripes. But the local symmetry and hole-spin dynamics will ultimately decide which kind of stripes is most stable. In the following, we consider some general properties of stripes with holedipoles as elementary building blocks.

Anti-phase. Let us consider a line-up of hole-dipoles along, say, $\hat{x}$-axis with the dipole orientations satisfying the optimal condition Eq. (39). Namely, $\left(\mathbf{e}_{k}\right)_{x} \equiv e_{x}$, $\left(\mathbf{e}_{k}\right)_{y} \equiv(-1)^{k} e_{y}$, where integer $k$ is an index labelling dipoles. Here we assume that each dipole has the same size of a dipole moment $|\mathbf{e}|$ with a minimal spacing $2 e_{x}$ between two dipoles. For a site far away from the $\hat{x}$-axis, the spin twist is then given by

$$
\begin{aligned}
\phi_{i} & =\sum_{k} \phi_{i}^{k}=\sum_{k} \frac{e_{x} y_{i k}-(-1)^{k} e_{y} x_{i k}}{r_{i k}^{2}} \\
& \simeq \sum_{k} \frac{e_{x} y_{i k}}{r_{i k}^{2}}=e_{x} \sum_{k=-\infty}^{+\infty} \frac{y_{i}}{\left(2 e_{x} k+x_{0}\right)^{2}+y_{i}^{2}} \\
& \simeq \frac{\pi}{2} \operatorname{sgn}\left(y_{i}\right),
\end{aligned}
$$

when $\left|y_{i}\right| \gg|\mathbf{e}| \sim a$. Here $y_{i k}=y_{i}$ and $x_{i k}=2 e_{x} k+x_{0}$ according to the definition.

Thus, an anti-phase ( $\pi$ phase shift) is found across the stripe with

$$
\Delta \phi=\phi_{y>0}-\phi_{y<0}=\pi .
$$

Namely, a stripe composed of hole-dipoles is topologically always an anti-phase domain wall of spins.

Metallic stripe. For Type A dipoles discussed in Sec. III, a minimal choice of $\mathbf{e}$ is $e_{x}=a$ and $e_{y}=0$, as shown in Fig. 4(a). If one simply line up these dipoles along the $\hat{x}$-axis, then the corresponding stripe is an anti-phase domain wall with quarter-filled holons

$$
n_{h}=\frac{1}{4}
$$

(i.e., one hole per two sites), as illustrated in Fig. 5(a).

Obviously the twists of spins due to the presence of hole-dipoles are all canceled out away from the stripe, such that the total potential energy is minimized. Without spin frustrations, two stripes will not gain additional 


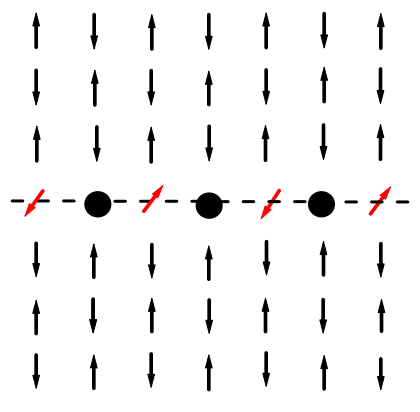

Metallic stripe from type A dipoles

(a)

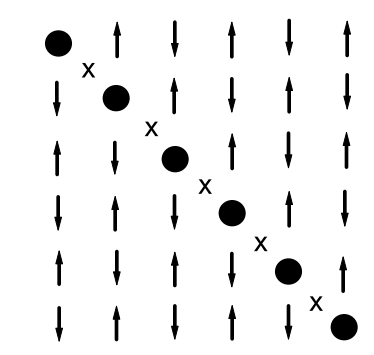

Diagonal stripe from type B dipoles

(b)

FIG. 5: Two kinds of stripes as composed of two elementary types of hole-dipoles shown in Fig. 4: (a) An anti-phase, quarter-filling, metallic stripe; (b) An anti-phase, insulating stripe.

energy by being close together and thus these stripes are also stable against a cluster formation. Furthermore, within each anti-phase stripe, the hole-dipoles will disassociate to gain additional kinetic energy. It is straightforward to see now holons can move along the stripe without causing additional frustrations, just like in the onedimensional (1D) $t-J$ model [5]. Here the flowing of the "river of charges" along the stripe will gain energy $\sim-0.9 t_{h}$ for each holon at quarter-filling. This gaining of the kinetic energy is in contrast to the energy cost for a hole-dipole escaping away from the stripe: $\mathcal{E}^{d} \sim J_{s}$ according to Eq.(28) 24]. The energy difference will stabilize such a metallic stripe of quarter-filling, at least in a local minimal sense. In literature, a metallic stripe based on the $t-J_{z}$ model has been discussed in Ref. [25]. The spectral density for a single hole in the stripe phase has been also studied in Ref. [26].

Based on the phase string picture, a metallic stripe discussed above can be regarded as a line which connects those branch-cuts between the starting and ending points of holons in Fig. 3. As emphasized before, a phase string is so singular that it can only be eliminated when one holon tightly tails another, which leads to a 1D line of charges rather than a pairing of holes. Physically it is the phase string effect that ultimately threads holes together in a metallic stripe.

As a natural extrapolation, one may picture a stripe liquid as composed of mesoscale stripes threaded by phase strings, after the melting of static stripes. Eventually, say at high enough temperature, the most elementary entities, i.e., hole-dipoles, should be also present, if spins are still ordered (Bose condensed). Experimentally, how to detect such mesoscale stripes or even individual hole-dipole objects is an interesting subject to further explore in the present framework.

Diagonal stripe. For a Type B dipole, its minimal dipole moment is shown in Fig. 4(b). Here one pole is located inside a plaquette while the pole of the holon is at a corner of the plaquette. Fig. 5(b) shows a natural stripe configuration composed of the minimal Type $\mathrm{B}$ dipoles in Fig. 4(b). It is a stripe along the diagonal direction, which remains as an anti-phase domain as the argument leading to Eq. (41) still holds here. But it becomes an insulating stripe instead, as holons cannot directly move along the stripe.

It has been argued that hole-dipoles of Type B may be more stable at low doping as it costs less superexchange energy. By forming a diagonal stripe, there is no obvious gain in the hole's kinetic energy, except for some virtual process. In a single hole-dipole case, such a virtual process may be understood as each holon continuously hops around and then back to its origin, leaving irreparable phase strings on the paths which result in a spin-dipole configuration. When these holes are lined up in the stripe configuration, the phase strings are canceled out each other during the virtual hopping process such that there is no spin twist left behind away from the stripe [Fig. 5]. So for both types of stripe in Fig. 5, the healing of the spin AF order away from the domain wall indicates an effective kinetic energy gain for virtual hole hopping out of the domain wall. Furthermore, holons gains additional kinetic energy in the metallic stripe, but the superexchange energy of the domain wall seems favored in the diagonal stripe, under a given number of holes.

One may envisage some other kinds of static stripes. A detailed analysis of which type of stripes is most energetically favorable in a given doping concentration is beyond the scope of the present paper, and will be explored elsewhere. Finally, we note that various charge orderings due to hole-hole long-range (dipolar and Coulomb) interactions have been also studied numerically in Ref. 
[27], based on a spin-density-wave, or itinerant approach. Our strong-couling (t-J type model) approach seems to provide a consistent picture concerning the formation of static stripes, though many details still need a further comparison.

\section{DISCUSSIONS}

In this paper, we have studied how holes behave in a spin ordered background at low doping. In a doped AF Mott insulator described by a derivative model of the $t-J$ Hamiltonian, known as the phase string model, the kinetic energy of doped holes gets strongly frustrated by the phase string effect. These holes induce nonlocal effects in the spin background and behave like dipoles. In contrast to the hole dipole picture of Shraiman and Siggia [16] based on the semiclassical treatment, a holon constitutes one of the two poles in the present configuration instead of sitting at the center of the dipole [16]. Consequently, hole dipoles are localized or self-trapped objects in space rather than mobile ones in the Shraiman-Siggia's case. Such a localization or self-trapping of a hole can be intuitively understood directly in terms of the irreparable phase string effect [19].

That the hole kinetic energy is strongly suppressed at low doping in the phase string model is in contrast to the slave-boson theories 9$]$ of the $t-J$ model. In the latter cases, holes are relatively free moving on various RVB backgrounds. The difference arises from the fact that in the bosonic RVB background [8], short-range $A F$ correlations are much stronger than in fermionic RVB theories, which are highly unfavorable to the hole motion.

Due to the frustrations of the hole kinetic energy, the dipole-dipole interaction dominates the low-energy, long-wavelength physics. Holes correlate with each other through such an interaction which, instead of leading to a real-space pairing of holes, causes an inhomogeneous instability - stripe instability with hole-dipoles collapsing into a 1D line. An anti-phase, quarter-filling metallic stripe can be regarded as a pile up of Type A dipoles, while an anti-phase, diagonal insulating stripe may be naturally considered as composed of Type B dipoles. Here the phase string effect provides the basic thread for forming stripes: holes tightly follow each other's paths in order to minimize the frustrations of the phase string effect and to release the kinetic energy.

Theoretically, stripes and stripes related issues have been studied intensively in literature [4], motivated by experimental discovery [28] of static stripes in curpates. In fact, insulating stripes as a mean-field solution was first obtained 29, 30, 31 in the Hubbard and $t-J$ models at low-doping even before the experiment. The present approach has gone beyond the mean-field approximation in dealing with the motion of doped holes in an antiferromagnet. It reveals that as a "renormalization" effect there does exist a residual long-range interaction for holes to "talk" to each other and to collectively form stripes. This approach provides a framework to further systematically investigate various properties associated with stripe phenomenon. Finally, in literature stripes as composed of ferromagnetic bonds beyond the $t-J$ type model have been previously proposed 32]. But the origin of the underlying physics as well as the consequence are different.

Some interesting questions remain to be answered. Firstly, if static stripes melt, are there dynamic stripes present at higher energy or higher temperature? If they exist, it is also a very challenge problem to mathematically describe such dynamic stripes of "finite sizes". Recently a $\mathrm{Z}_{2}$ gauge theory of "stripe fractionalization" has been proposed by Nussinov and Zaanen [33] based on a "topological" order of the sublattice parity. The $\mathrm{Z}_{2}$ phase string depicted in Fig. 1(a) indeed originates from the change of the sublattice parity of spins due to the hole hopping. But the phase string model of Eqs. (2) and (3) is not simply a $\mathrm{Z}_{2}$ gauge theory because the original building blocks are holons and spinons, not in a stripe fractionalization description.

Of course, in the end the holons are actually confined in the spinon condensed phase and elementary objects become hole-dipoles. A mesoscale stripe structure may be expected as remaining clusters at high energy or temperature, threaded by phase strings, which should eventually break into hole-dipoles. But we note that the above dipole picture and stripe instability are all discussed when spinons are Bose condensed. When such a condensation is gone at high temperature or larger doping, the dipole and stripe pictures are no longer meaningful in this framework.

Secondly, what is the relation between superconductivity and stripe phases? So far we have not explored the possibility of superconducting condensation in the spinon condensed phase, where stripes present. In the phase string model, superconducting condensation happens when holons are Bose condensed [6, 12]. It can occur at higher doping where no spinon condensation is present [12]. Thus, superconducting phase and stripe phase seem not necessarily concomitant in this framework. Nevertheless, the origin of superconductivity and that of stripes can all be attributed to kinetic-energydriven mechanisms. Since the phase string effect plays the concrete role of kinetic-energy frustrations, various low-temperature phases including stripes, superconductivity, and even the pseudogap phenomenon may be regarded as different ways in reducing kinetic energies in such a system 7].

\section{Acknowledgments}

We acknowledge stimulating discussions with $\mathrm{N}$. P. Ong which partially motivated the present work. This work is partially supported by NSFC grant nos. 90103021, 10247002, and 10204004. 
[1] P.W. Anderson, Science, 235, 1196 (1987).

[2] S. Trugman, Phys. Rev. B37, 1597 (1988).

[3] D. N. Sheng, Y. C. Chen, and Z. Y. Weng, Phys. Rev. Lett. 77, 5102 (1996).

[4] For a review, see, E.W. Carlson, V.J. Emery, S.A. Kivelson, and D. Orgad, cond-mat/0206217 and references therein.

[5] Z. Y. Weng, D. N. Sheng, Y. C. Chen, and C. S. Ting, Phys. Rev. B55, 3894 (1997).

[6] Z. Y. Weng, D. N. Sheng, and C. S. Ting, Phys. Rev. Lett. 80, 5401 (1998).

[7] S. P. Kou and Z. Y. Weng, cond-mat/0206560 (2000).

[8] S. Liang, B. Doucot and P. W. Anderson, Phys. Rev. Lett. 61, 365 (1988).

[9] Z. Zou and P. W. Anderson, Phys. Rev. B37, 627 (1988); N. Nagaosa and P. A. Lee, Phys. Rev. Lett. 64, 2450 (1990); X. G. Wen and P. A. Lee, Phys. Rev. Lett. 76, 503 (1996).

[10] D. P. Arovas and A. Auerbach, Phys. Rev. B 38, 316 (1988).

[11] S. Sarker, C. Jayaprakash, H. R. Krishnamurthy, and M. Ma, Phys. Rev. B 40, 5028 (1989); D. Yoshioka, J. Phys. Soc. Jpn 58, 32 (1989).

[12] Y. Zhou, V.N. Muthukumar, and Z.Y. Weng, cond-mat/0208017 to appear in Phys. Rev. B (2003).

[13] J.A. Verges et al., Phys. Rev. B43, 6099 (1991); M. Berciu and S. John, Phys. Rev. B59, 15143 (1999);

[14] C. Timm and K.H. Bennemnn, Phys. Rev. Lett. 84, 4994 (2000).

[15] R. Eder, Phys. Rev. B 45, 319 (1992); P. Wróbel and R. Eder, Phys. Rev. B 58, 15160 (1998).

[16] B. Shraiman and E. Siggia, Phys. Rev. Lett. 61, 467 (1988); B. Shraiman and E. Siggia, Phys. Rev. Lett. 62, 1564 (1989).

[17] D.M. Frenkel and W. Hanke, Phys. Rev, B 42, 6711
(1990).

[18] E. Manousakis, Nuc. Phys. B 21, 689 (1991).

[19] Z.Y. Weng, V.N. Muthukumar, D.N. Sheng, and C.S. Ting, Phys. Rev. B63, 075102 (2001).

[20] A. Aharony et al., Phys. Rev. Lett. 60, 1330 (1988).

[21] L. I. Glazman and A. S. Ioselevich, Z. Phys. B80, 133 (1990).

[22] V. Cherepanov, et al. cond-mat/9808235 I. Y. Korenblit, et al., cond-mat/9709056

[23] K.S.D. Beach, R.J. Gooding, cond-mat/0001095

[24] Note that the self-energy of a hole-dipole also includes a hopping energy as the holon confined to the dipole may still hop around locally according to $H_{h}$ [Eq.(2)]. Rigorously speaking, one needs to consider both hopping energy and the confining potential energy Eq.(28) in determining the stability.

[25] A.L. Chernyshev, A.H. Castro Neto, A.R. Bishop, Phys. Rev. Lett. 84, 4922 (2000).

[26] P. Wróbel and R. Eder, Phys. Rev. B 62, 4048 (2000).

[27] B.P. Stojkovic, Z.G. Yu, A.R. Bishop, A.H. Castro Neto, and N. Gronbech-Jensen, Phys. Rev. Lett. 82, 4679 (1999); Phys. Rev. B 62, 4353 (2000).

[28] J. M. Tranquada et al., Nature 375 (1995) 561; J. M. Tranquada et al., Phys. Rev. B 54 (1996) 7489.

[29] J. Zaanen and O. Gunnarsson, Phys. Rev. B40, 7391 (1989).

[30] H. J. Schulz, Phys. Rev. Lett., 64, 1445(1990).

[31] K. Machida, Physica C158, 192 (1989); M. Kato, K. Machida, H. Nakanishi and M. Fujita, J. Phys. Soc. Jpn 59, 1047 (1990).

[32] N.M. Salem and R. J. Gooding, Europhys. Lett. 35, 603 (1996).

[33] Z. Nussinov and J. Zaanen, cond-mat/0209437 J. Zaanen and Z. Nussinov, cond-mat/0209441 